\section{(2) OPEN ACCESS}

\title{
A novel faecal Lachnoclostridium marker for the non- invasive diagnosis of colorectal adenoma and cancer
}

\author{
Jessie Qiaoyi Liang, ${ }^{1}$ Tong Li, ${ }^{1}$ Geicho Nakatsu, ${ }^{1}$ Ying-Xuan Chen, ${ }^{2}$ Tung On Yau, ${ }^{1}$ \\ Eagle Chu, ${ }^{1}$ Sunny Wong (D) ,' Chun Ho Szeto, ${ }^{1}$ Siew C Ng (D) ,' Francis K L Chan (D) ,' \\ Jing-Yuan Fang, ${ }^{2}$ Joseph J Y Sung, ${ }^{1}$ Jun Yu (D) ${ }^{1}$
}

\begin{abstract}
- Additional material is published online only. To view please visit the journal online (http://dx.doi.org/10.1136/ gutjnl-2019-318532).

${ }^{1}$ Institute of Digestive Disease and Department of Medicine and Therapeutics, State Key Laboratory of Digestive Disease, Li Ka Shing Institute of Health Sciences, CUHK Shenzhen Research Institute, The Chinese University of Hong Kong, Shatin, Hong Kong

2Division of Gastroenterology, Shanghai Jiaotong University School of Medicine Renii Hospital, Shanghai Institute of Digestive Disease, Shanghai, China
\end{abstract}

\section{Correspondence to}

Dr Jun Yu, Department of Medicine and Therapeutics, The Chinese University of Hong Kong, Hong Kong, Hong Kong; junyu@cuhk.edu.hk

Dr Jessie Qiaoyi Liang; jessieqy@cuhk.edu.hk

Received 17 February 2019 Revised 11 November 2019 Accepted 14 November 2019 Published Online First 27 November 2019

\section{ABSTRACT \\ Objective There is a need for early detection of} colorectal cancer (CRC) at precancerous-stage adenoma. Here, we identified novel faecal bacterial markers for diagnosing adenoma.

Design This study included 1012 subjects (274 CRC 353 adenoma and 385 controls) from two independent Asian groups. Candidate markers were identified by metagenomics and validated by targeted quantitative PCR.

Results Metagenomic analysis identified ' $m 3$ ' from a Lachnoclostridium sp., Fusobacterium nucleatum (Fn) and Clostridium hathewayi $(\mathrm{Ch})$ to be significantly enriched in adenoma. Faecal $m 3$ and $F n$ were significantly increased from normal to adenoma to $C R C(p<0.0001$, linear trend by one-way ANOVA) in group I $(n=698)$, which was further confirmed in group II $(n=313 ; p<0.0001)$. Faecal $m 3$ may perform better than $F n$ in distinguishing adenoma from controls (areas under the receiver operating characteristic curve (AUROCs) $m 3=0.675 \mathrm{vs}$ $F n=0.620, p=0.09$ ), while $F n$ performed better in diagnosing CRC (AUROCs $F n=0.862$ vs $m 3=0.741$, $\mathrm{p}<0.0001$ ). At $78.5 \%$ specificity, $m 3$ and $F n$ showed sensitivities of $48.3 \%$ and $33.8 \%$ for adenoma, and $62.1 \%$ and $77.8 \%$ for CRC, respectively. In a subgroup tested with faecal immunochemical test (FIT; $n=642)$, m3 performed better than FIT in detecting adenoma (sensitivities for non-advanced and advanced adenomas of $44.2 \%$ and $50.8 \%$ by $m 3$ (specificity $=79.6 \%$ ) vs $0 \%$ and $16.1 \%$ by FIT (specificity=98.5\%)). Combining with FIT improved sensitivity of $m 3$ for advanced adenoma to $56.8 \%$. The combination of $m 3$ with $F n, C h$, Bacteroides clarus and FIT performed best for diagnosing CRC ( ppecificity $=81.2 \%$ and sensitivity $=93.8 \%$ ).

Conclusion This study identifies a novel bacterial marker $m 3$ for the non-invasive diagnosis of colorectal adenoma.

\section{INTRODUCTION}

Colorectal cancer (CRC) is one of the most common malignancies worldwide. ${ }^{1}$ A higher incidence of CRC has been observed in more developed regions than less developed regions, and an increased incidence of CRC is believed to have attributed to changes in diet. ${ }^{12}$ Recent evidences have shown that an altered microbiome environment in the gut is associated with colorectal tumourigenesis. Abnormality in the composition of the gut microbiota has been implicated as a potentially important

\section{Significance of this study}

What is already known on this subject?

- Early detection of colonic adenomas and cancer can facilitate the successful treatment and significantly reduces the incidence of colorectal cancer (CRC).

- Molecular markers for adenoma, especially nonadvanced adenoma, is limited.

What are the new findings?

- A new gene marker from a Lachnoclostrium sp., labelled as $m 3$, was identified to be enriched in faecal samples of patients with adenoma by metagenomic analysis.

- $m 3$ showed the best performance in diagnosing adenoma in two independent Asian groups of 1012 subjects by quantitative $P C R$, which is superior to currently available stool-based tests.

- Combination of $m 3$ with faecal immunochemical test (FIT) improved diagnostic sensitivity from $50.8 \%$ to $56.8 \%$ (specificity $79.6 \%$ ) for advanced adenoma, while combination of $m 3$ with other bacterial markers $(F n, C h, B C$ ) and FIT showed good diagnostic performance for CRC (specificity $=81.2 \%$ and sensitivity $=93.8 \%$ ).

How might it impact on clinical practice in the foreseeable future?

- $m 3$ is a novel stool-based non-invasive biomarker for patients with adenoma and CRC.

aetiological factor in the initiation and progression of CRC. ${ }^{3}$ With the widespread application of metagenomic analyses in the investigation of intestinal microbiota, an increasing number of bacteria have been identified to be positively associated with CRC. $^{4-7}$ Recent basic research has established a critical function for the intestinal microbiota ${ }^{8}$ and specific bacterial species, such as Fusobacterium nucleatum $(F n)^{9-11}$ and Peptostreptococcus anaerobius, ${ }^{12}$ in promoting colorectal tumourigenesis. Bacteria such as $\mathrm{Fn}^{13}{ }^{13}$ Clostridium symbiosum ${ }^{14}$ and species within the genera Parvimonas, Porphyromonas and Parabacteroides ${ }^{15}$ have been shown to be potential markers for the diagnosis of patients with CRC. However, current knowledge on biomarkers for colorectal adenoma detection is limited. 
Early detection of cancer can facilitate successful treatment. Endoscopic removal of colorectal adenomas, precursors of most CRCs, significantly reduces the risk of CRC. Early detection of adenomas is thus important for decreasing CRC morbidity and mortality. The most widely used non-invasive stool test is the faecal immunochemical test (FIT), which shows unsatisfying sensitivities for CRC (0.79 (95\% CI 0.69 to 0.86); differed greatly among various studies) and is not sensitive for adenoma. ${ }^{16}$ Sensitivity of FIT for advanced adenoma varied from $6 \%$ to $56 \%$, with screening studies involving cohort sizes over 8000 all showing sensitivities of less than $28 \% .{ }^{17}$ Therefore, identification of molecular markers that improve the diagnostic sensitivity for adenoma is warranted.

Using metagenomic analysis to compare the faecal microbiome of patients with CRC and healthy subjects, we identified 20 bacterial gene marker candidates that may serve as noninvasive biomarkers for CRC. ${ }^{4}$ We further showed that stoolbased bacteria could serve as non-invasive diagnostic biomarkers for CRC by targeted quantification using quantitative PCR (qPCR). ${ }^{13}$ We showed that $F n$ was a good marker for CRC, and combination with three others (Clostridium hathewayi $(C h)$, undefined ' $m 7$ ' and Bacteroides clarus $(B c)$ ) could further improve the diagnostic performance of $F n$. However, the diagnostic performance of these bacterial gene markers for adenoma was limited. In this study, we identified and evaluated the utility of a new Lachnoclostridium gene marker (labelled as ' $m 3$ ') for the diagnosis of colorectal adenoma. The diagnostic performance of $m 3$, comparing with and in combination with other bacterial gene markers and FIT, was tested in 1012 subjects from two independent groups.

\section{RESULTS}

Identification of ' $m 3^{\prime}$ ' from a Lachnoclostridium species as a potential biomarker for colorectal neoplasm

To investigate whether our previously identified 20 bacterial gene markers for $\mathrm{CRC}^{4}$ may also serve as biomarkers for adenoma, we analysed their abundances in our in-house metagenomics data from 589 Asian subjects (184 CRC, 185 adenoma and 220 control subjects) (online supplementary table S1). Among them, the marker labelled as ' $m 3$ ', which was not assignable to any known species at the time of the previous discovery study, ${ }^{4}$ was found to be significantly enriched in patients with CRC and adenoma as compared with control subjects, as well

\section{A Metagenome-seq}
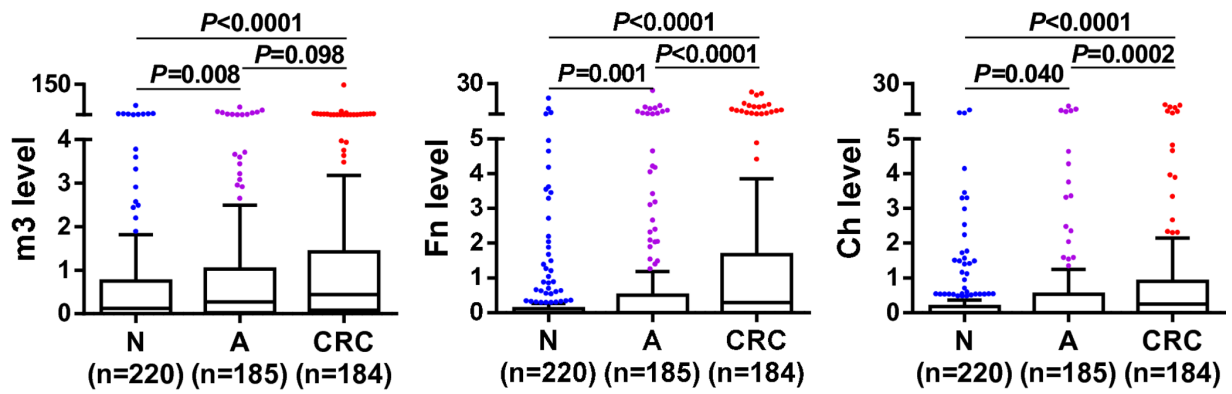

B
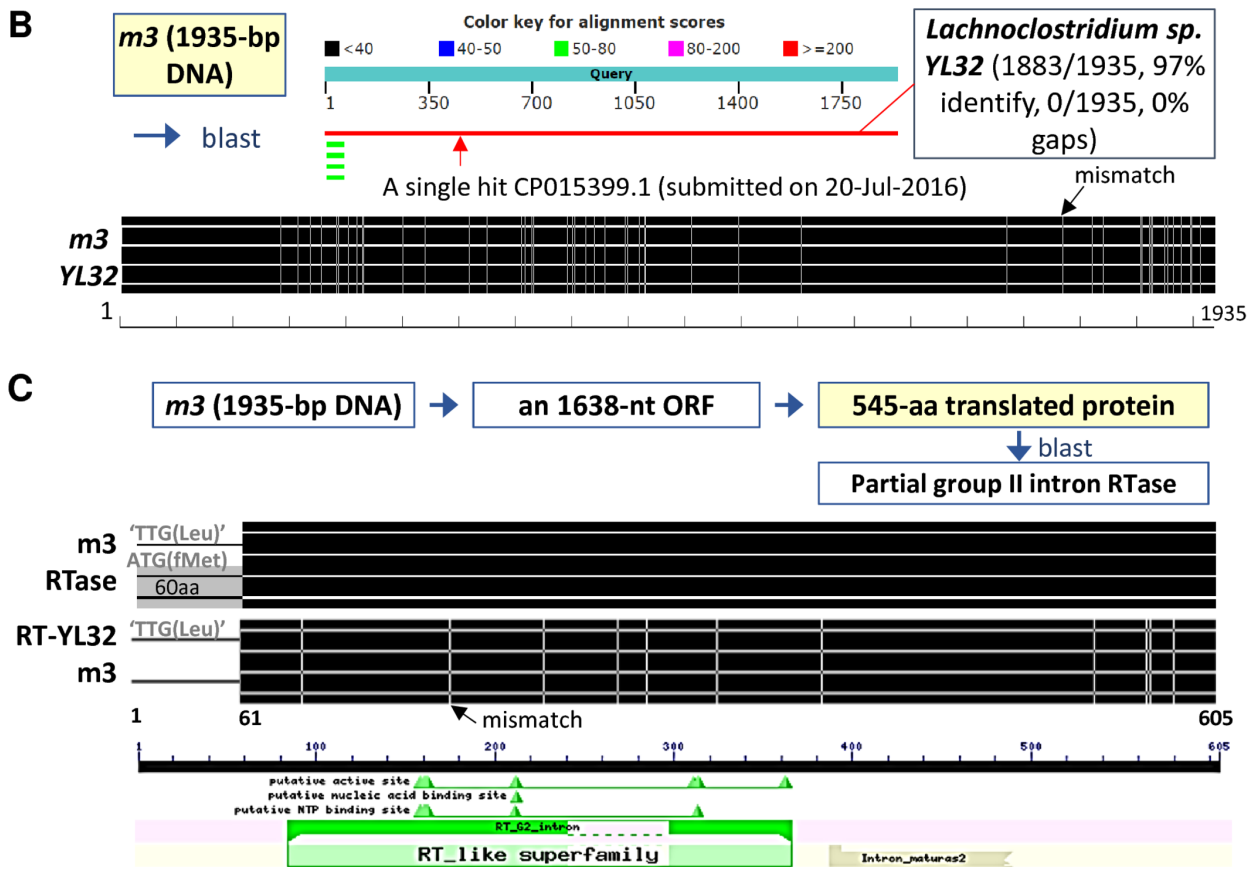

Figure 1 Identification and characterisation of $m 3$. (A) Metagenome sequencing identified $m 3$, as well as Fusobacterium nucleatum (Fn) and Clostridium hathewayi (Ch), to be significantly increased in faecal samples of patients with adenoma. (B) DNA sequence of $m 3$ showed high similarity to Lachnoclostridium sp. YL32. (C) $m 3$ encodes a putative reverse transcriptase (RTase) that maps to a group II intron RTase, lacking the first 60 amino acids but retaining the RTase conserved domain. A, adenoma; CRC, colorectal cancer; N, normal control. 
as two previously verified CRC markers ( $F n$ and $C h$ ) (figure $1 \mathrm{~A}$; other gene markers in online supplementary figure 1). A blast search for the $1935 \mathrm{nt} m 3$ sequence in the non-redundant nucleotide collection of NCBI identified Lachnoclostridium sp. YL32, a new species with genome sequence deposited in GenBank in July 2016 (accession no. CP015399). $m 3$ and Lachnoclostridium sp. YL32 shared 97\% (1883/1935) DNA sequence similarity (figure 1B). The $m 3$ DNA contains a $1638 \mathrm{nt}$ open reading frame (nt 298-1935), encoding a putative 545 aa protein with $100 \%$ sequence similarity to a group II RTase (GenBank accession no. WP 055650193). Although $m 3$ protein lacks the first 60 aa due to 'TTG' codon instead of 'ATG' at the corresponding translation start site, it retains the RTase conserved domain (figure 1C). The corresponding sequence in Lachnoclostridium sp. YL32 genome also encodes a partial group II RTase, showing 98\% $(534 / 545)$ sequence similarity with $m 3$-RTase and the group II RTase. We further analysed the abundance of Lachnoclostridium sp. YL32 genome based on the Prokka-annotated protein coding gene sequences in our in-house metagenomics data. The result showed that Lachnoclostridium sp. YL32 was significantly increased in adenoma but to a less extent in CRC as compared with control subjects (online supplementary figure 2A). Therefore, the candidate gene marker $m 3$ may belong to Lachnoclostridium species close to Lachnoclostridium sp. YL32.

\section{Validation of $m 3$ as a novel faecal biomarker for colorectal adenoma by $\mathrm{qPCR}$}

We further quantitatively examined the abundance of $m 3$ in an enlarged group of stool samples from Hong Kong Chinese by using our previously established duplex-qPCR platform. ${ }^{13}$ The results showed that faecal $m 3$ level was significantly higher in patients with adenoma $(n=207)$ versus control subjects $(n=288)$, and was significantly higher in patients with CRC $(n=203)$ versus control subjects or patients with adenoma (all $p<0.0001$ by multiple comparison). There was a significant linear trend of $m 3$ increasing from control to adenoma to cancer $(\mathrm{p}<0.0001$, one-way ANOVA) (figure 2A). Interestingly, $m 3$ level was similar
A

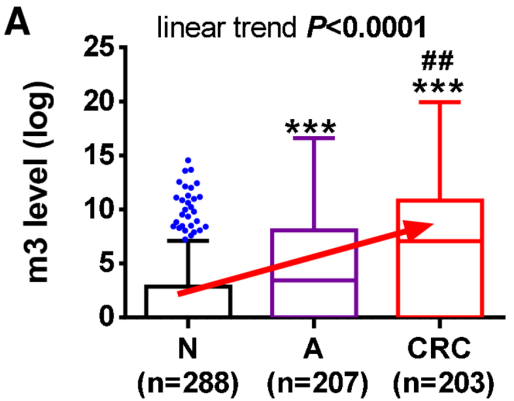

B

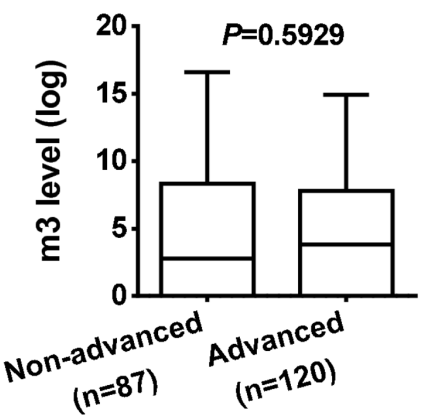

E

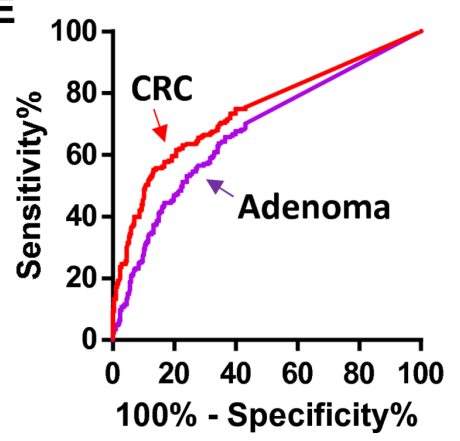

C

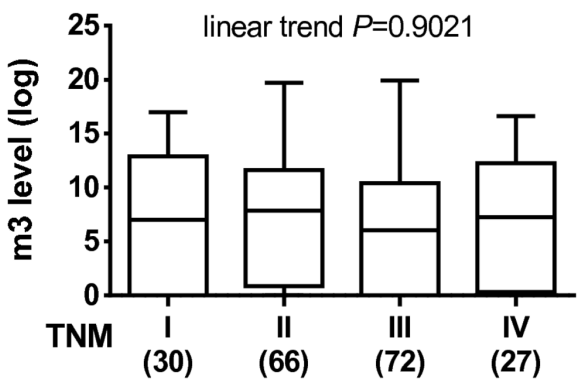

D

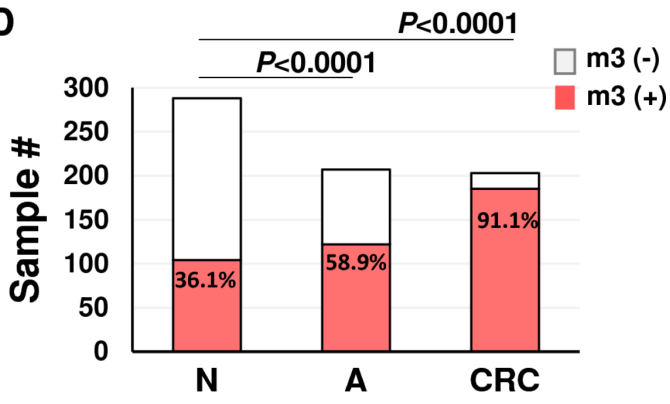

Figure 2 Quantitative detection of faecal $m 3$ in the diagnosis of patients with colorectal cancer (CRC) and adenoma. (A) Relative abundance of $m 3$ in faecal samples differed significantly between healthy control subjects $(N, n=288)$, patients with adenoma $(A, n=207)$ and patients with $C R C$ $(\mathrm{n}=203) .{ }^{* *} \mathrm{p}<0.0001$ as compared with $\mathrm{N} ;{ }^{* \#} \mathrm{p}<0.001$ as compared with $\mathrm{A}$. (B) No significant difference in faecal abundance of $m 3$ was observed between non-advanced and advanced adenomas. (C) No difference in faecal abundance of $m 3$ was observed among patients with CRC of different tumour-node-metastasis (TNM) stages. (D) Occurrence rates of $m 3$ was significantly higher in patients with adenoma compared with control subjects, and highest in patients with CRC. (E) Receiver operating characteristic (ROC) curves and diagnostic performance of $m 3$ in discriminating patients with CRC and adenoma from control subjects, respectively. AUROC, area under ROC. 

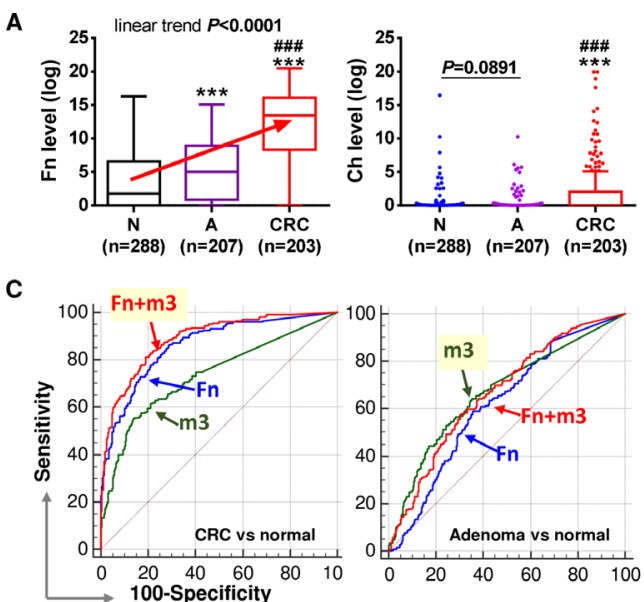

B

\begin{tabular}{|c|c|c|c|c|c|c|}
\hline \multicolumn{2}{|c|}{ Variable } & AUC & SE $^{\mathbf{a}}$ & $\mathbf{9 5 \%} \mathbf{C l}^{\mathbf{b}}$ & $\mathbf{z}^{\text {stat. }}$ & $\boldsymbol{P}$ \\
\hline \multirow{2}{*}{ CRC } & $\boldsymbol{F n}$ & $\mathbf{0 . 8 6 3}$ & 0.017 & 0.829 to 0.892 & 21.682 & $<\mathbf{0 . 0 0 0 1}$ \\
\cline { 2 - 7 } & $\boldsymbol{C h}$ & $\mathbf{0 . 6 4 7}$ & 0.021 & 0.603 to 0.690 & 7.157 & $<\mathbf{0 . 0 0 0 1}$ \\
\hline \multirow{2}{*}{ Adenoma } & $\boldsymbol{F n}$ & $\mathbf{0 . 6 2 0}$ & 0.025 & 0.576 to 0.663 & 4.796 & $<\mathbf{0 . 0 0 0 1}$ \\
\cline { 2 - 7 } & $\boldsymbol{C h}$ & 0.530 & 0.018 & 0.485 to 0.574 & 1.668 & 0.0954 \\
\hline
\end{tabular}

D

\begin{tabular}{|c|c|c|c|c|c|c|}
\hline \multirow{2}{*}{ Variabl } & \multicolumn{3}{|c|}{ CRC } & \multicolumn{3}{|c|}{ Adenom } \\
\hline & $F n$ & m3 & m3\&Fn & $F n$ & m3 & \\
\hline & 0.863 & 41 & 891 & 0.620 & 75 & 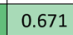 \\
\hline$g_{t}$ & & & & 663 & & 713 \\
\hline$P(v s)$ & $<0.0001$ & & 0.0001 & 0. & & .89 \\
\hline$P(v s$ & & 0.0001 & & & & 1.000 \\
\hline ensit & & & $.8 \%$ & $3 \%$ & $3 \%$ & 4.6 \\
\hline ecci & 78. & & & $78.5 \%$ & $\% \%$ & 0.2 \\
\hline & & & & & 5. & \\
\hline
\end{tabular}

Figure 3 Comparison and combination of bacterial markers for non-invasive diagnosis of colorectal cancer (CRC) and adenoma. (A) Relative abundances of Fusobacterium nucleatum (Fn), Clostridium hathewayi $(\mathrm{Ch})$ in faecal samples of control subjects, patients with adenoma and patients

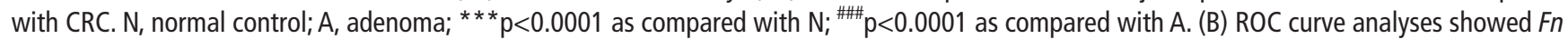
could discriminate adenoma and CRC from controls, while Ch could discriminate CRC but not adenoma from controls. (C) Comparison of ROC curves of $F n, m 3$ and their combination. (D) Diagnostic performances of $F n, m 3$ and their combination. Fn performed better than $m 3$ in diagnosing CRC, and $m 3$ was superior to $F n$ in diagnosing adenoma. Combination with $F n$ improved the diagnostic performance of $m 3$ for CRC but not for adenoma.

between non-advanced and advanced adenomas (figure 2B). Similarly, $m 3$ level showed no significant change across tumournode-metastasis staging in patients with CRC (figure 2C). The occurrence rate of faecal $m 3$ was significantly higher in patients with adenoma as compared with control subjects, and highest in patients with CRC (both $\mathrm{p}<0.0001$ vs control subjects; figure 2D). Receiver operating characteristic (ROC) curve analysis showed that $m 3$ could significantly discriminate CRC and adenoma patients from control subjects, with areas under ROC (AUROCs) of 0.741 and 0.675 for CRC and adenoma, respectively (both $\mathrm{p}<0.0001$; figure $2 \mathrm{E}$ ). At specificity of $78.5 \%, \mathrm{m3}$ showed sensitivities of $62.1 \%$ for CRC and $48.3 \%$ for adenoma; the accuracies were $71.7 \%$ and $65.9 \%$ for distinguishing patients with CRC and adenoma from control subjects, respectively. These results demonstrated that $m 3$ may serve as a new stoolbased biomarker to assist the non-invasive diagnosis of CRC and adenoma.

\section{$m 3$ performs better than other bacterial markers ( $F n$ and $C h$ ) in diagnosing colorectal adenoma}

As $F n$ and $C h$ were also identified to be significantly increased in patients with adenoma by metagenome sequencing, we further examined the levels of $F n$ and $C h$ by qPCR and compared their diagnostic performances with $m 3$. Results confirmed that, similar to $m 3$, the relative faecal abundance of $F n$ was significantly elevated in patients with adenoma compared with control subjects, and highest in patients with CRC, with a significant linear trend of increase during CRC development (all $\mathrm{p}<0.0001$; figure $3 \mathrm{~A}$ ). However, faecal $\mathrm{Ch}$ was not significantly increased in patients with adenoma as compared with control subjects by qPCR ( $p>0.05)$. Ch was significantly enriched in patients with CRC compared with patients with adenoma and control subjects (both $\mathrm{p}<0.0001$, figure $3 \mathrm{~A}$ ). ROC curve analyses also showed that both $\mathrm{Fn}$ and $\mathrm{Ch}$ performed well in diagnosing CRC, but only $F n$ could significantly distinguish patients with adenoma from control subjects $(\mathrm{p}<0.0001$; figure $3 \mathrm{~B})$. The abundances of both $F n$ and $m 3$ were not associated with gender, CRC staging, lesion location or body mass index. Multivariate analysis showed that $F n$ and $m 3$ were significantly associated with CRC and adenoma diagnosis, as well as $F n$ with age (table 1 ). Although $F n$ performed better than $m 3$ in CRC diagnosis (AUROC of $F n=0.863$ vs $m 3=0.741 ; \mathrm{p}<0.0001), m 3$ may work better than Fn for adenoma diagnosis as shown by comparison of ROC curves (AUROCs: $m 3=0.675$ vs $F n=0.620, \mathrm{p}=0.09$; figure $3 \mathrm{C}, \mathrm{D}$ ). As single diagnostic factors at specificity of $78.5 \%$, Fn discriminated patients with CRC from control subjects with a sensitivity of $77.8 \%$ and accuracy of $78.2 \%$ (vs $62.1 \%$ and $71.7 \%$, respectively, by $m 3)$. At specificity of $78.5 \%, m 3$ discriminated patients

Table 1 Correlations between faecal marker abundances and clinical characteristics

\begin{tabular}{|c|c|c|c|c|c|c|c|c|}
\hline \multirow{3}{*}{$\begin{array}{l}\text { Markers } \\
\text { Variable }\end{array}$} & \multicolumn{4}{|c|}{ Univariate } & \multicolumn{4}{|c|}{ Multivariate } \\
\hline & \multicolumn{2}{|l|}{ Fn } & \multicolumn{2}{|l|}{$m 3$} & \multicolumn{2}{|l|}{ Fn } & \multicolumn{2}{|l|}{$m 3$} \\
\hline & Coef & $P$ value & Coef & $P$ value & Coef & $P$ value & Coef & $P$ value \\
\hline Gender & -0.348 & 0.443 & -0.143 & 0.706 & & & & \\
\hline Diagnosis & 2.768 & $<0.0001$ & 1.535 & $<0.0001$ & 2.597 & $<0.0001$ & 1.472 & $<0.0001$ \\
\hline Pre/post-colonoscopy & -0.181 & 0.729 & -0.735 & 0.095 & & & & \\
\hline CRC staging & 0.173 & 0.689 & -0.227 & 0.620 & & & & \\
\hline Lesion location & -0.153 & 0.862 & -0.201 & 0.830 & & & & \\
\hline
\end{tabular}

BMI, body mass index; CRC, colorectal cancer. 


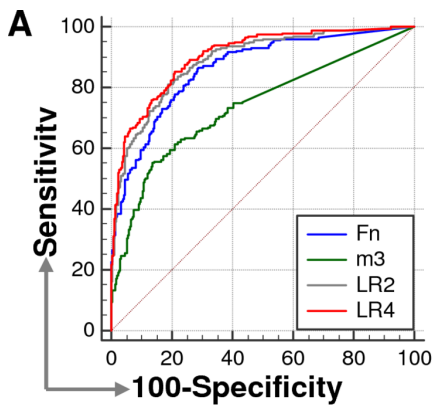

\begin{tabular}{|c|c|c|c|c|c|}
\hline For CRC & AUC & SE & $95 \% \mathrm{Cl}$ & Markers & $\boldsymbol{P}$ \\
\hline $\mathrm{Fn}$ & 0.863 & 0.0167 & 0.829 to 0.892 & $\mathrm{Fn}$ & \multirow{6}{*}{ 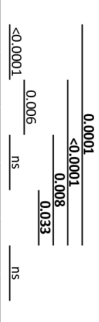 } \\
\hline $\mathrm{m} 3$ & 0.741 & 0.0227 & 0.699 to 0.779 & m3 & \\
\hline LR2 & 0.891 & 0.0147 & 0.860 to 0.917 & $\mathrm{Fn}, \mathrm{m} 3$ & \\
\hline LR3 & 0.895 & 0.0144 & 0.865 to 0.921 & $\mathrm{Fn}, \mathrm{m} 3, \mathrm{Bc}$ & \\
\hline LR4 & 0.907 & 0.0133 & 0.877 to 0.931 & $\mathrm{Fn}, \mathrm{m} 3, \mathrm{Bc}, \mathrm{Ch}$ & \\
\hline LR5 & 0.907 & 0.0133 & 0.877 to 0.931 & $\mathrm{Fn}, \mathrm{m} 3, \mathrm{Bc}, \mathrm{Ch}, \mathrm{m} 7$ & \\
\hline
\end{tabular}
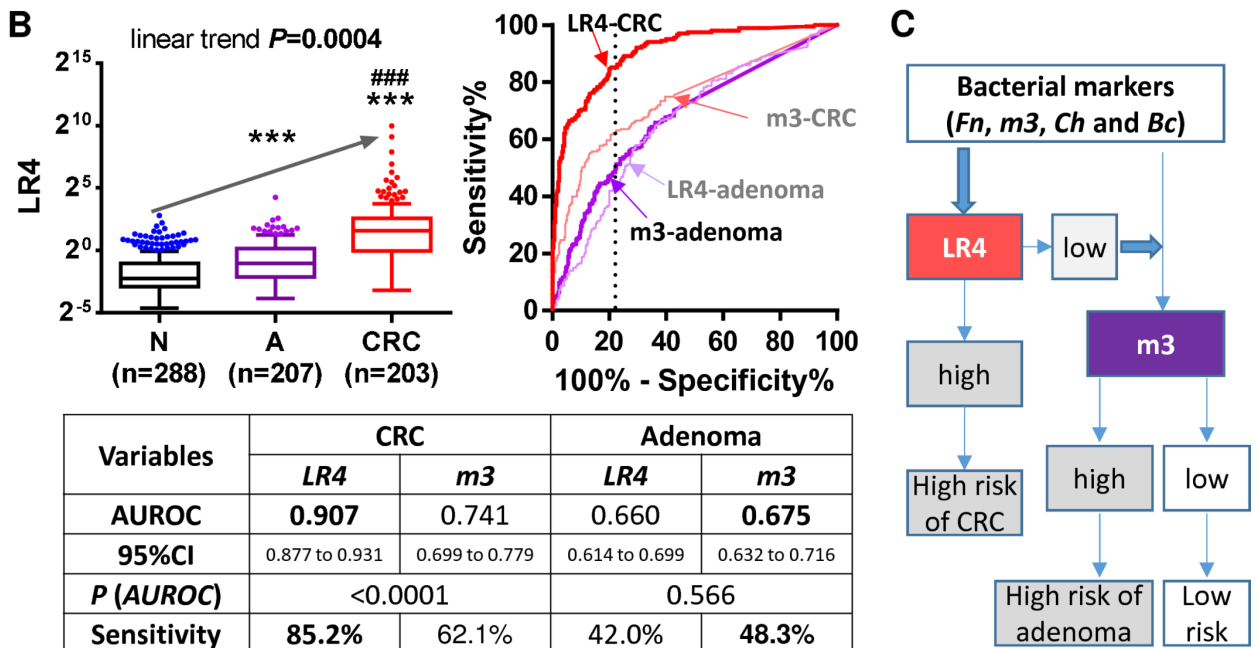

\begin{tabular}{|c|c|c|c|c|}
\hline \multirow{2}{*}{ Variables } & \multicolumn{2}{|c|}{ CRC } & \multicolumn{2}{c|}{ Adenoma } \\
\cline { 2 - 5 } & $\mathbf{L R 4}$ & $\mathbf{m 3}$ & $\mathbf{L R 4}$ & $\mathbf{m 3}$ \\
\hline AUROC & $\mathbf{0 . 9 0 7}$ & 0.741 & 0.660 & $\mathbf{0 . 6 7 5}$ \\
\hline $\mathbf{9 5 \% C l}$ & 0.877 to 0.931 & 0.699 to 0.779 & 0.614 to 0.699 & 0.632 to 0.716 \\
\hline $\boldsymbol{P}$ (AUROC) & \multicolumn{2}{|c|}{$<0.0001$} & \multicolumn{2}{c|}{0.566} \\
\hline Sensitivity & $\mathbf{8 5 . 2 \%}$ & $62.1 \%$ & $42.0 \%$ & $\mathbf{4 8 . 3} \%$ \\
\hline Specificity & $\mathbf{8 0 . 2} \%$ & $78.5 \%$ & $80.2 \%$ & $\mathbf{7 8 . 5} \%$ \\
\hline Accuracy & $\mathbf{8 2 . 3 \%}$ & $71.7 \%$ & $64.2 \%$ & $\mathbf{6 5 . 9 \%}$ \\
\hline
\end{tabular}

Figure 4 Combination of four markers for olorectal cancer (CRC) and $m 3$ alone for adenoma. (A) Receiver operating characteristic (ROC) curve analysis of combination of the five bacterial markers of interest showed that combination of $F n, m 3, C h$ and $B c$ by a logistic regression (LR) model worked best for CRC diagnosis. Shown p values are by comparison ROC curves. (B) Level of the combination of $F n, m 3, C h$ and $B c$ (LR4) in faecal samples and comparison of its diagnostic performance with $m 3$. N, normal control; $\mathrm{A}$, adenoma; ${ }^{* * *} \mathrm{p}<0.0001$ as compared with $\mathrm{N}$; ${ }^{* \# \#} \mathrm{p}<0.0001$ as compared with A. (C) Proposed strategy for the application of $F n, m 3, C h$ and $B c$ in the diagnosis of $C R C$ and adenoma.

with adenoma from control subjects with a sensitivity of $48.3 \%$ and accuracy of $65.9 \%$ (vs $33.8 \%$ and $59.8 \%$, respectively, by $\mathrm{Fn}$ ) (figure 3C,D). Interestingly, combination of $F n$ and $m 3$ by a logistic regression model significantly improved their individual diagnostic performances for CRC (AUROC $=0.891, \mathrm{p}=0.003 \mathrm{vs}$ $\mathrm{F} n$ ) but not for adenoma (AUROC $=0.671, \mathrm{p}=0.89 \mathrm{vs} \mathrm{m} 3$ ). These results demonstrate that $m 3$ is a potential good diagnostic biomarker especially for adenoma.

\section{Combination with other bacterial markers ( $F n, B C$ and $C h$ ) increases the diagnostic performance of $m 3$ for CRC, while $m 3$ alone works best for adenoma}

As we have previously reported bacterial markers $F n, C h, B c$ and $m 7$ for diagnosis of CRC, we further tested the performance of the combination of these markers with $\mathrm{m} 3$ for CRC. The results showed that combination of $F n, m 3, C h$ and $B c$ by a logistic regression model performed best in diagnosing CRC, with an AUROC of 0.907 (all $\mathrm{p}<0.05$ as compared with combinations of fewer markers by comparison of ROC curves; figure 4A). At specificity of $80.2 \%$, combination of $F n, m 3, C h$ and $B c$ showed a sensitivity of $85.2 \%$ and accuracy of $82.3 \%$ for CRC. Although combination of $F n, m 3, C h$ and $B c$ showed significantly increased score in patients with adenoma as compared with control subjects, its diagnostic performance for adenoma was not better than $m 3$ (AUROC $=0.660$ vs $m 3=0.675, \mathrm{p}=0.086$; figure $4 \mathrm{~B})$. Therefore, combination of $F n, m 3, C h$ and $B c$ may serve as a novel tool for the non-invasive diagnosis of CRC, while $m 3$ alone may be applied for further detection of adenoma (figure 4C).

\section{Verification of diagnostic performance of bacterial markers in a second independent group}

We further tested the bacterial markers in a second independent group of 313 samples from Shanghai China (71 CRC, 145 adenoma and 97 controls). Results showed that both $F n$ and $m 3$ were significantly increased in patients with adenoma compared with control subjects, and further increased in patients with cancer, with a significant linear trend of increase from control to adenoma to cancer by multiple comparison $(p<0.0001$; figure $5 \mathrm{~A}$ ). Using the same logistic regression model established in the Hong Kong group, the combined score of the four markers $(F n, m 3, C h$ and $B c)$ also showed a significant increase during the normal-adenoma-CRC sequence $(\mathrm{p}<0.0001)$. Fn performed better than $m 3$ in discriminating CRC from controls although not significantly (AUROCs for CRC: $F n=0.776$ vs $m 3=0.759$ ), and the four-marker combination showed the best performance in diagnosing CRC (AUROC $=0.830$, sensitivity $=77.5 \%$ and specificity $=75.3 \%$; figure $5 \mathrm{~B}$ ). $m 3$ performed better than $F n$ and the four-marker combination in distinguishing patients with adenoma from controls although not significantly (AUROCs: $m 3=0.662, \quad F n=0.616$ and four-marker $=0.652$ ), and $m 3$ showed a sensitivity of $51.0 \%$ and specificity of $75.3 \%$ for 


\section{A Group II - Shanghai}
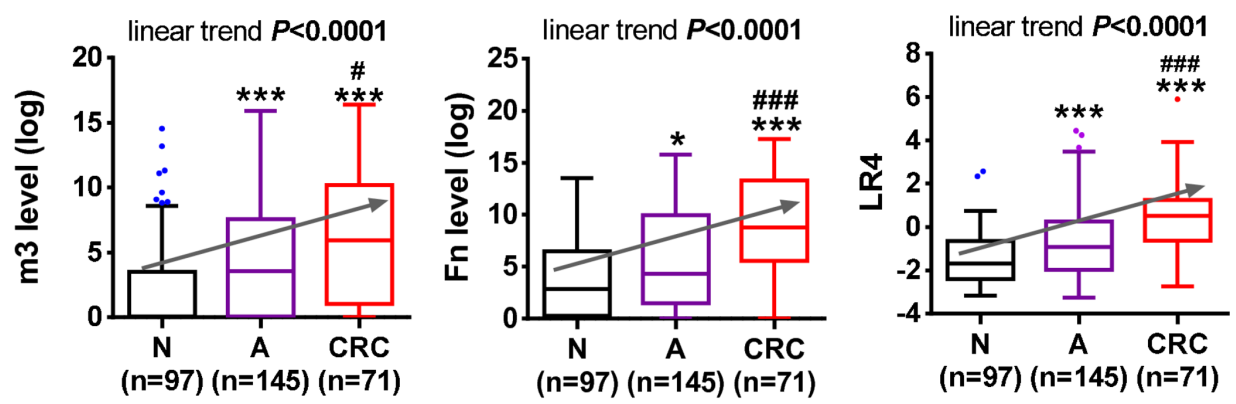

B
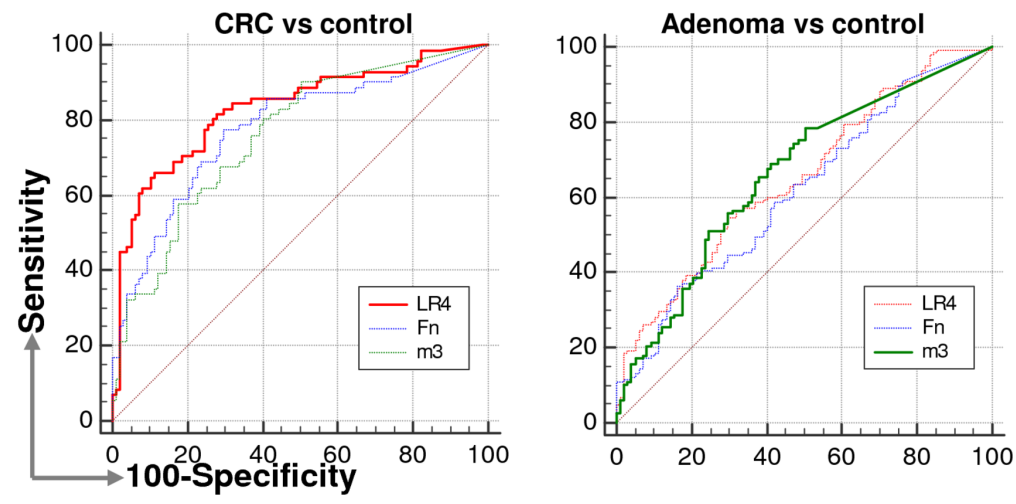

\begin{tabular}{|c|c|c|c|c|c|c|}
\hline \multirow{2}{*}{ Variables } & \multicolumn{3}{|c|}{ CRC } & \multicolumn{3}{c|}{ Adenoma } \\
\cline { 2 - 7 } & $\boldsymbol{F n}$ & $\boldsymbol{m 3}$ & $\mathbf{L R 4}$ & $\boldsymbol{F n}$ & $\mathbf{m 3}$ & $\mathbf{L R 4}$ \\
\hline AUROC & 0.776 & 0.759 & $\mathbf{0 . 8 3 0}$ & 0.616 & $\mathbf{0 . 6 6 2}$ & 0.652 \\
\hline $\mathbf{9 5 \%} \% \mathbf{C l}$ & 0.705 to 0.837 & 0.687 to 0.822 & 0.765 to 0.884 & 0.552 to 0.678 & 0.599 to 0.722 & 0.589 to 0.712 \\
\hline $\boldsymbol{P}(\mathbf{0 . 5})$ & $<0.0001$ & $<0.0001$ & $<0.0001$ & 0.0014 & $<0.0001$ & $<0.0001$ \\
\hline $\boldsymbol{P}$ (vs $\mathbf{m} \mathbf{3})$ & 0.7475 & - & 0.0470 & 0.3475 & - & 0.7821 \\
\hline $\boldsymbol{P}$ (vs $\boldsymbol{F n})$ & - & 0.7475 & 0.0465 & - & 0.3475 & 0.1305 \\
\hline Sensitivity & $59.2 \%$ & $62.0 \%$ & $\mathbf{7 7 . 5 \%}$ & $35.9 \%$ & $\mathbf{5 1 . 0 \%}$ & $42.8 \%$ \\
\hline Specificity & $83.5 \%$ & $75.3 \%$ & $\mathbf{7 5 . 3 \%}$ & $83.5 \%$ & $\mathbf{7 5 . 3 \%}$ & $75.3 \%$ \\
\hline Accuracy & $73.2 \%$ & $69.6 \%$ & $\mathbf{7 6 . 2 \%}$ & $55.0 \%$ & $\mathbf{6 0 . 7 \%}$ & $55.8 \%$ \\
\hline
\end{tabular}

Figure 5 Validation of bacterial markers in diagnosing colorectal cancer (CRC) and adenoma in a second independent group of faecal samples. (A) Relative faecal abundances of $F n$ and $m 3$ and level of the combination of $F n, m 3, C h$ and $B c$ (LR4) in patients with CRC and adenoma compared with control subjects of the second group. $N$, normal control; $A$, adenoma; ${ }^{*} p<0.05$ and ${ }^{* * *} p<0.0001$ as compared with $N$; ${ }^{\#}<<0.05$ and ${ }^{\# \#} p<0.001$ as compared with A. (B) Comparison of ROC curves and diagnostic performances of $F n, m 3$ and LR4.

adenoma (figure 5B). These results further confirm the diagnostic values of the four bacterial markers for CRC and $m 3$ for adenoma.

\section{Combination with FIT improves the diagnostic ability of bacterial markers for CRC}

To compare the diagnostic performance of bacterial markers with the most widely used non-invasive stool test in CRC screening, FIT was performed in a subgroup of 642 samples from Hong Kong (178 CRC, 204 adenoma and 260 controls). The CRC detection rate by combination of $\mathrm{Fn}, \mathrm{m3}, \mathrm{Ch}$ and $\mathrm{Bc}$ $(84.7 \%)$ was significantly higher than $m 3$ alone $(61.8 \%)$ or FIT (71.3\%) (both $\mathrm{p}<0.01)$. The combination of FIT with fourmarker showed the best performance in detecting CRC, with a sensitivity of $93.8 \%$ and specificity of $81.2 \%$ (figure $6 \mathrm{~A}$ ). The four-marker showed higher sensitivities than FIT for stages I-III cancers but not late stage IV. Combination of the four-marker and FIT showed significantly increased sensitivities than FIT for stages I-III cancers, and also elevated the detection rate for stage
IV cancers (figure 6B). These results demonstrate that the bacterial marker panel is superior to FIT for detection of stages I-III $\mathrm{CRC}$, and their combination further improves the non-invasive diagnosis of CRC.

\section{$m 3$ performs significantly better than FIT in diagnosing adenoma}

In the subgroup of 204 adenoma (86 non-advanced and 118 advanced) cases with FIT results, $m 3$ alone (sensitivity $=48.0 \%$ ) showed a significantly higher detection rate than FIT (9.3\%) and the four-marker $(42.2 \%)$ (both $\mathrm{p}<0.001)$. Combination of $m 3$ with FIT showed the best diagnostic performance for adenoma, with a sensitivity of $51.5 \%$ and specificity of $78.1 \%$ (figure $6 \mathrm{~A}$ ). FIT failed to detect any non-advanced adenoma $(0 / 86,0 \%)$ and detected $16.1 \%(19 / 118)$ of the advanced adenoma. On the other hand, $m 3$ showed no difference in the detection between non-advanced and advanced adenomas (figures $2 \mathrm{~B}$ and $6 \mathrm{C}$ ), and the detection rates of $m 3$ were significantly higher for both nonadvanced (44.2\%) and advanced (50.8\%) adenomas than FIT 
A

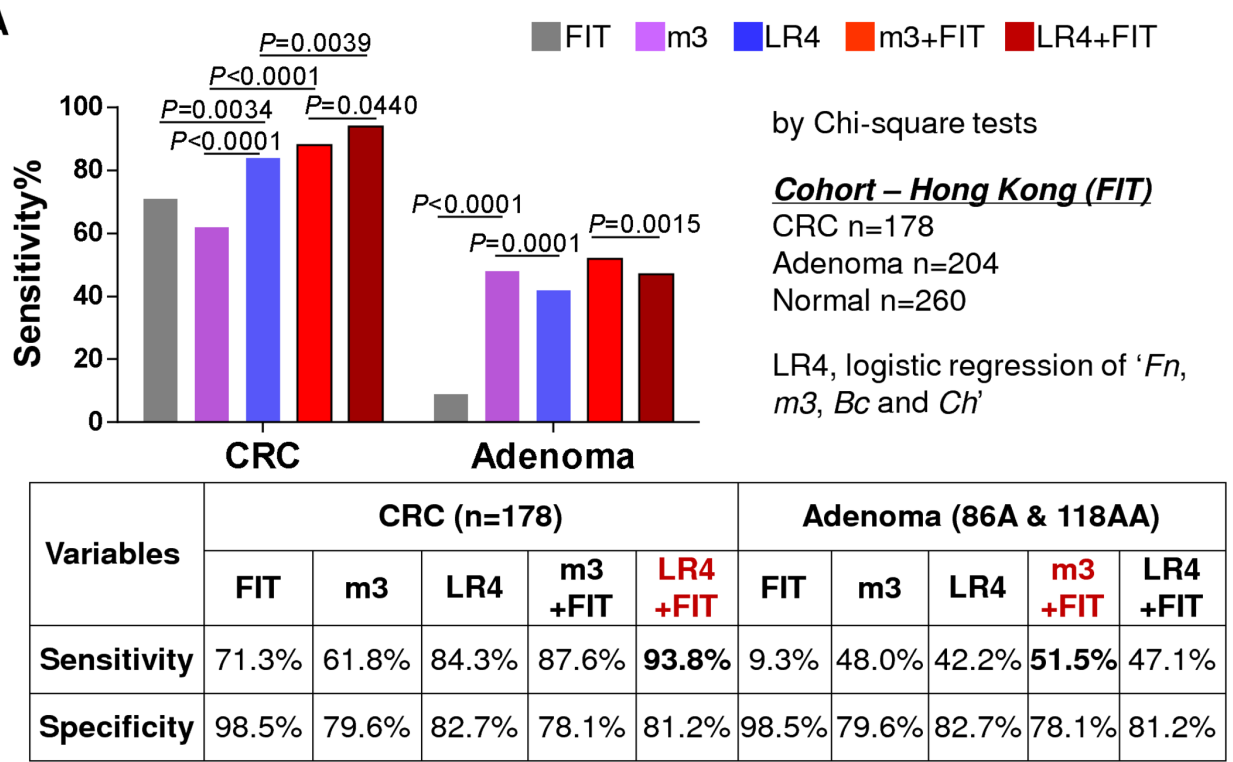

B

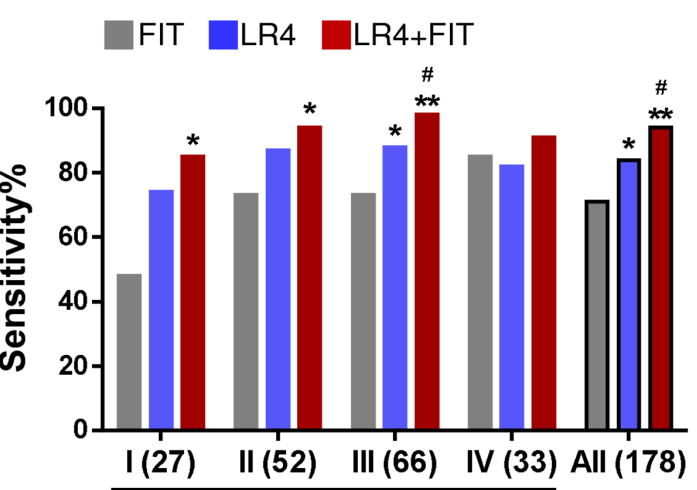

C $\quad$ FIT $m 3 \quad m 3+F I T$

TNM-stage subset

${ }^{*} P<0.05$ and ${ }^{*} P<0.001$ vs FIT

${ }^{\#} P<0.05$ and ${ }^{\#} P<0.001$ vs LR4

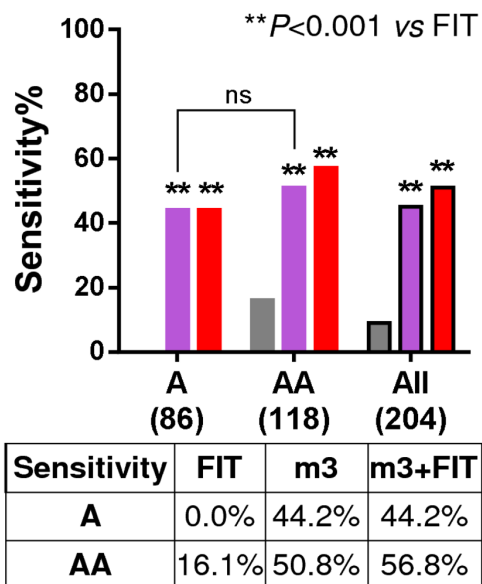

Figure 6 Comparison and combination of bacterial markers with faecal immunochemical test (FIT). (A) Comparison of sensitivity and specificity of FIT, $m 3$, combination of four makers ( $F n, m 3, C h$ and $B c$; LR4) and combination of bacterial markers with FIT in a subgroup of Hong Kong samples. LR4 combined with FIT performed best for colorectal cancer (CRC) detection, while $m 3$ combined with FIT performed best for detecting adenoma. (B) Comparison of the sensitivities of FIT, LR4 and their combination in detecting CRC according to tumour-node-metastasis (TNM) stage subsets. (C) Comparison of the sensitivities of FIT, $m 3$ and their combination in detecting non-advanced and advanced adenomas. All comparison of sensitivities was conducted by $\chi^{2}$ tests. $A$, non-advanced adenoma; $A A$, advanced adenoma.

(both $\mathrm{p}<0.001$; figure 6C). Combination with FIT increased the sensitivity of $m 3$ for advanced adenoma to $56.8 \%$. These results demonstrate that $m 3$ alone shows good performance for stoolbased detection of adenoma.

\section{DISCUSSION}

In this study, we screened a previously identified panel of CRCassociated gene markers in patients with CRC or adenoma compared with control subjects by metagenomics analysis. Focusing on the candidate gene markers that were significantly changed in patients with adenoma as compared with control subjects, we further validated their application values in noninvasive diagnosis of adenoma and CRC by qPCR. We demonstrated a faecal bacterial marker $m 3$ that is useful for adenoma detection and at the mean time devised a new panel of faecal bacterial markers $(m 3+F n+C h+B c)$ to achieve improved diagnostic capacity for CRC as compared with markers reported in our previous studies. ${ }^{413}$

$m 3$ is a well-assembled DNA sequence (1935 bp) from shotgun sequencing data. As $m 3$ DNA is long enough and distinct from DNA polymerase genes of other micro-organisms, with a single specific hit to Lachnoclostridium sp. YL32 with high score (97\% identify), it is reliable to conclude that the host bacterium of ' $m 3$ ' belong to the bacterial genus Lachnoclostridium. We further analysed the abundances of known Lachnoclostridium genomes in our in-house metagenomics data, with 160 strains from GenBank and 27 species from ChocoPhlAn pangenome database (online supplementary figure $2 \mathrm{~A}, \mathrm{~B}$ ). We found stepwise increase from control to adenoma to cancer in some species, such as Clostridium (C.) aldenense, C. bolteae, C. citroniae and C. clostridioforme (online supplementary figure 2C), demonstrating the potential of Lachnoclostridium species 
in discriminating patients with colorectal neoplasm from control subjects. Lachnoclostridium is a newly defined genus under the highly polyphyletic class Clostridia, ${ }^{18}$ with an increasing number of new species identified from human gut microbiota in recent few years, such as Lachnoclostridium (L.) edouardi, ${ }^{19} \mathrm{~L}$. pacaense ${ }^{20}$ and L. touaregense. ${ }^{21}$ The Lachnoclostridium species carrying $m 3$ and its roles in colorectal tumourigenesis warrant further characterisation in future studies.

We have reported that combination of $F n, B c, C h$ and the undefined ' $m 7$ ' showed good diagnostic performance for CRC. ${ }^{13}$ Comparison of ROC curves showed that combination of ' $F$, $B c, C h$ and $m 3$ ' (AUROC $=0.907$ (0.877 to 0.931)) showed an increased AUROC than the combination of ' $\mathrm{F} n, \mathrm{Bc}$, Ch and $m 7$ ' (AUROC $=0.892$ (0.856 to 0.921)) in our Hong Kong group and in Shanghai group (AUROCs: 0.830 (0.765 to 0.884 ) with $m 3$ vs 0.795 (0.705 to 0.867$)$ with $m 7$ ). These results suggest the impact of $m 3$ on improving other bacterial markers for the noninvasive diagnosis of CRC.

$F n$ is prevalently detected in human CRC, with important roles in the initiation and progression of CRC. Fn level in colonic adenoma and adenocarcinoma tissues was found to be $>10-100$ times higher than normal colonic mucosa, ${ }^{10}$ demonstrating $F n$ accumulation may occur at an early stage of colonic tumourigenesis. However, there is disagreement about the relationship between $F n$ and colorectal adenoma. ${ }^{22} F n$ was found to be enriched in cancerous versus matched normal tissues, but not significantly higher in adenoma versus normal tissues in a European cohort. ${ }^{23}$ Similarly, faecal abundance of $F n$ was found to be strongly associated with CRC but not adenoma in a German cohort. ${ }^{24}$ Although we observed a significant increase of faecal $F n$ in patients with adenoma compared with control subjects, the diagnostic value of $F n$ for adenoma is not as good as $m 3$, and combination with $F n$ could not improve the diagnostic performance of $m 3$ for adenoma.

We have showed that the gram-negative bacterium $B c^{25}$ was significantly decreased in patients with CRC as compared with healthy subjects and thus could help improve diagnostic specificity. The gram-positive bacterium $C h$, which participates in glucose metabolism using carbohydrates as fermentable substrates to produce acetate, ethanol, carbon dioxide and hydrogen ${ }^{26}$ was significantly increased in patients with CRC compared with healthy subjects. However, faecal abundances of $B c$ and $C h$ showed no differences between patients with adenoma and control subjects.

On the other hand, $m 3$ is superior to other bacterial markers in discriminating patients with adenoma from control subjects according to our results from two independent Chinese groups, although its diagnostic capacity for CRC is not as good as Fn. FIT only detected $16.1 \%$ advanced adenoma and none of nonadvanced adenoma. The multitarget stool DNA test approved by the US Food and Drug Administration, which combines mutant and methylated DNA markers and a FIT, shows sensitivities of $42.4 \%$ for advanced adenoma and $17.2 \%$ for non-advanced adenoma. ${ }^{27}$ Although the sensitivity of $m 3(48.3 \%)$ is still low for adenoma, $m 3$ showed no significant difference in the detection between advanced and non-advanced adenomas, with sensitivities of $50.8 \%$ and $44.2 \%$, respectively. Therefore, $m 3$ may outperform all other available stool-based tests in detecting nonadvanced adenoma. Moreover, combination with FIT improved the detection rate of $\mathrm{m} 3$ for advanced adenoma from $50.8 \%$ to $56.8 \%$.

Some of the faecal samples were collected after colonoscopy, with $40.6 \%, 36.2 \%$ and $40.4 \%$ in control, adenoma and cancer groups, respectively $(\mathrm{p}=0.577)$. However, these post-colonoscopy samples were collected at least 1 month after colonoscopy when gut microbiome should have recovered to baseline. ${ }^{28}$ Furthermore, we have adjusted for confounding effects of sample collection before/after colonoscopy during marker discovery. ${ }^{4}$ There was no significant difference in $m 3$ level between pre-colonoscopy and post-colonoscopy adenoma samples by qPCR or metagenome sequencing. There were also no difference in $F n, m 3$ or the four-marker combination between pre-colonoscopy and post-colonoscopy samples of the control, adenoma or CRC groups (online supplementary figure 3). Therefore, the markers involved in this study may not be affected by colonoscopic/bowel-prep status, given enough time for gut microbiome to recover after colonoscopy. Although age and gender differed significantly among the groups, inclusion of age and gender in the logistic regression model did not affect the ROC curves for CRC and adenoma significantly (online supplementary figure 4).

Recent studies of CRC have identified a large number of faecal microbial markers, and attempts to combine such markers from shotgun metagenomics data showed good diagnostic performance. ${ }^{6} 2930$ Our recent meta-analysis of multicohort metagenomics data, covering 526 samples from Chinese, Austrian, American, and German and French cohorts, identified seven CRC-enriched bacterial species showing an AUROC of 0.8 in discriminating patients with CRC from control subjects, which was increased to 0.88 when the clinical data were added. ${ }^{6}$ Application of direct shotgun metagenomics to diagnosis is not costefficient due to cumbersome experimental procedure and heavy computing workload. Targeted detection of identified microbial marker candidates based on shotgun metagenomics for clinical application is a more promising strategy. Based on our bacterial gene markers identified by metagenomics investigation, quantification of four bacterial gene markers by qPCR shows an AUROC of 0.907 for CRC diagnosis in this study. However, as the true performance of the markers cannot be established from these case-control samples, future validation is required in large sample cohorts representative of the CRC screening populations. We have also reported for the first time that faecal CRC-enriched virome and mycobiome biomarkers distinguished CRC from controls with AUROCs of 0.802 and 0.93, respectively. ${ }^{29} 30$ The application of these viral and fungal markers to non-invasive diagnosis of CRC by targeted quantification needs further exploration.

In conclusion, we identified a novel bacterial marker $m 3$, from a Lachnoclostridium species, for the non-invasive diagnosis of colorectal adenoma. $m 3$ is superior to other bacterial markers and currently available stool-based tests for adenoma detection.

\section{METHODS}

\section{Metagenomic marker gene sequence analysis}

Metagenomic sequencing data from 589 Hong Kong Chinese subjects (184 CRC, 185 adenoma and 220 control subjects) from our previous study were analysed ${ }^{29}$ which included the discovery cohort of 74 CRC and 54 controls for the identification of the 20 CRC-related markers. ${ }^{4}$ Raw faecal shotgun metagenomic sequences were quality-trimmed and decontaminated as described previously. ${ }^{29}$ Low complexity subsequences of bacterial genes were hard-masked with the DUST program and indexed using the Burrows-Wheeler Aligner (BWA; V.0.7.17) to create the gene database for short read alignment. ${ }^{31} 32$ Postquality control sequences in FASTQ format were mapped against the BWA database with maximal exact match (mem) algorithm and default parameters of penalty scoring. Histograms of aligned 
sequence coverage were reported using the 'genomecov' module of BEDTools suite (V.2.27.0). ${ }^{33}$ Mean sequence coverage table of metagenomic samples was constructed by computing summed products of coverage depth and base-pair fraction of marker gene length for positional features in input BAM files. Multiple group comparison of clinical phenotype was performed by pairwise Wilcoxon's rank-sum tests, and $\mathrm{p}$ values were corrected by Benjamini-Hochberg step-up procedure. We then derived average weighted contribution (AWC) scores to estimate differential genomic enrichment and depletion of Lachnoclostridium species using marker gene sequences originating from the ChocoPhlAn pangenome database $(\mathrm{V} .293)^{34}$ as well as Prokkaannotated protein coding gene sequences representing 160 Lachnoclostridium genomes at all assembly levels from the NCBI GenBank (release 234.0; accessed 16 Oct 2019). ${ }^{35}$ The AWC of species $i$ with gene set $j$ to phenotype $k$ was computed as follows:

$$
\mathrm{AWC}_{i j k}=\frac{\sum_{j \in k} \mathrm{NE}_{i j}-\mathrm{ND}_{i j}}{\mathrm{~N}_{i j}^{2}},
$$

where $\mathrm{NE}_{i j}$ (or $\mathrm{ND}_{i j}$ ) is the total count of significant enrichment (or depletion) of a genomic sequence in gene set $j$ for species $i$ in a one-versus-all comparative statistical analysis of clinical phenotype $k$ at $5 \%$ false discovery rate, respectively. $\mathrm{N}_{i j}$ denotes the number of gene sequences of species $i$.

\section{Human faecal sample collection}

Faecal samples $(n=1012)$ were collected from two independent groups of subjects, including group I-Hong Kong (698 subjects: 203 CRC, 207 adenoma and 288 normal controls) at the Prince of Wales Hospital, the Chinese University of Hong Kong between 2009 and 2014 and group II-Shanghai (313 subjects: 71 CRC, 145 adenoma and 97 normal controls) at Renji Hospital, Shanghai Jiaotong University between 2014 and 2018 (detailed clinical characteristics in online supplementary table S2). Subjects recruited for faecal sample collection included individuals presenting symptoms such as change of bowel habit, rectal bleeding, abdominal pain or anaemia, and asymptomatic individuals aged 50 or above undergoing screening colonoscopy as in our previous metagenomic study. ${ }^{4}$ Samples were collected before or 1 month after colonoscopy, when gut microbiome should have recovered to baseline. ${ }^{28}$ The exclusion criteria were (1) use of antibiotics within the past 3 months, (2) on a vegetarian diet, (3) had an invasive medical intervention within the past 3 months and (4) had a history of any cancer or inflammatory disease of the intestine. Subjects were asked to collect stool samples in standardised containers at home and store the samples in their home freezer at $-20^{\circ} \mathrm{C}$ immediately. Frozen samples were then delivered to the hospitals in insulating polystyrene foam containers and stored at $-80^{\circ} \mathrm{C}$ immediately until further analysis. Patients were diagnosed by colonoscopic examination and histopathological review of any biopsies taken.

\section{DNA extraction, design of primers and probes and qPCR}

DNA extraction, design of primer and probe sequences and qPCR amplifications on an ABI QuantStudio sequence detection system were conducted as our previous description. ${ }^{13}$ Primer and probe sequences specifically targeting $m 3$ are as following: forward 5'-AATGGGAATGGAGCGGATTC-3'; reverse 5'-CCTGCACCAGCTTATCGTCAA-3'; probe 5'AAGCCTGCGGAACCACAGTTACCAGC- $3^{\prime}$. Primer and probe sequences targeting other bacterial gene markers and 16s rDNA internal control are as in our previous study. ${ }^{13}$ Each probe carried a $5^{\prime}$ reporter dye FAM (6-carboxy fluorescein) or VIC (4,7,2'-trichloro-7'-phenyl-6-carboxyfluorescein) and a $3^{\prime}$ quencher dye TAMRA (6-carboxytetramethyl-rhodamine). Primers and hydrolysis probes were synthesised by Invitrogen (Carlsbad, CA). PCR amplification specificity was confirmed by direct Sanger sequencing of the PCR products or by sequencing randomly picked TA clones. Relative abundance of each marker was calculated by using delta $\mathrm{Cq}$ method as compared with internal control and shown as Log value of "*10e6+1'.

\section{Faecal immunochemical test}

A subgroup of Hong Kong samples $(n=642 ; 178$ CRC, 118 advanced adenoma, 86 non-advanced adenoma and 260 control subjects) were examined by FIT using the automated quantitative OC-Sensor test (Eiken Chemical, Japan). The quantitative OC-Sensor test was performed as our previous description, ${ }^{36}$ with a positive cut-off value equivalent to a concentration of $100 \mathrm{ng}$ of haemoglobin per millilitre.

\section{Statistical analyses}

Values were all expressed as mean $\pm \mathrm{SD}$ or median (IQR) as appropriate. The differences in bacterial abundances were determined by Mann-Whitney U test. One-way ANOVA multiple comparison with test for linear trend was used to evaluate the changes of marker levels during disease progression (from control to adenoma to cancer). Simple and multiple regression analyses were used to estimate the association between marker levels and factors of interest. Occurrence rates between different groups and sensitivities by different markers were analysed using the $\chi^{2}$ test. Combination of multiple biomarkers was performed by applying logistic regression model to obtain values for estimating the incidence of CRC as compared with controls. The scores of the combination of four markers were calculated as follows: LR4 $=$ Power $\left(2,\left(\alpha+\beta_{1} X_{1}+\beta_{2} X_{2}+\beta_{3} X_{3}+\beta_{4} X_{4}\right)\right)$, where $\alpha$ represented the intercept, $\beta$ represented the regression coefficients and $\mathrm{X}$ represented the levels of the corresponding markers. ROC curves were used to evaluate the diagnostic value of bacterial markers/models in distinguishing CRC/adenoma and controls. Pairwise comparison of ROC curves was performed using a nonparametric approach. ${ }^{37}$ The best cut-off values were determined by ROC analyses that maximised the Youden index ( $\mathrm{J}=$ Sensitivity + Specificity -1$).{ }^{38}$ All tests were done by GraphPad Prism V.5.0 (GraphPad Software, San Diego, CA) or MedCalc Statistical Software V.18.5 (MedCalc Software bvba, Ostend, Belgium; http://www.medcalc.org; 2018). A p value $<0.05$ was taken as statistical significance.

Contributors Study conception and design: QL, JY. Development of methodology: QL. Acquisition of data: TL, Y-XC, TOY, GN, EC, SW, SCN, FKLC, Y-YF. Analysis and interpretation of data: QL. Writing, review and/or revision of the manuscript: QL, FKLC, JJYS, JY. Administrative, technical or material support: QL, JY. Study supervision: QL, JJYS, JY.

Funding This project was supported by HRMF research fellowship scheme (02160037), China MOST fund (2016YFC1303200), Science and Technology Program Grant Shenzhen (JCYJ20170413161534162), National Key R\&D Program of China (2017YFE0190700, 2018YFC135000, 2018YFC1315004), National Natural Science Foundation of China (81773000) and Shenzhen Virtual University Park Support Scheme to CUHK Shenzhen Research Institute.

Competing interests None declared.

Patient consent for publication Obtained.

Ethics approval The study was approved by the Clinical Research Ethics Committee of the Chinese University of Hong Kong and the Ethics Committee of Renji Hospital, Shanghai Jiaotong University.

Provenance and peer review Not commissioned; externally peer reviewed.

Data availability statement All data relevant to the study are included in the article or uploaded as online supplementary information. 
Open access This is an open access article distributed in accordance with the Creative Commons Attribution Non Commercial (CC BY-NC 4.0) license, which permits others to distribute, remix, adapt, build upon this work non-commercially, and license their derivative works on different terms, provided the original work is properly cited, appropriate credit is given, any changes made indicated, and the use is non-commercial. See: http://creativecommons.org/licenses/by-nc/4.0/.

ORCID iDs

Sunny Wong http://orcid.org/0000-0002-3354-9310

Siew C Ng http://orcid.org/0000-0002-6850-4454

Francis K L Chan http://orcid.org/0000-0001-7388-2436

Jun Yu http://orcid.org/0000-0001-9239-2416

\section{REFERENCES}

1 Allemani C, Matsuda T, Di Carlo V, et al. Global surveillance of trends in cancer survival 2000-14 (CONCORD-3): analysis of individual records for 37513025 patients diagnosed with one of 18 cancers from 322 population-based registries in 71 countries. Lancet 2018;391:1023-75.

2 The Lancet. GLOBOCAN 2018: counting the toll of cancer. Lancet 2018;392:985

3 Irrazábal T, Belcheva A, Girardin SE, et al. The multifaceted role of the intestina microbiota in colon cancer. Mol Cell 2014;54:309-20.

4 Yu J, Feng Q, Wong SH, et al. Metagenomic analysis of faecal microbiome as a tool towards targeted non-invasive biomarkers for colorectal cancer. Gut 2017;66:70-8

5 Nakatsu G, Li X, Zhou H, et al. Gut mucosal microbiome across stages of colorectal carcinogenesis. Nat Commun 2015;6:8727.

6 Dai Z, Coker 00, Nakatsu G, et al. Multi-cohort analysis of colorectal cancer metagenome identified altered bacteria across populations and universal bacterial markers. Microbiome 2018;6.

7 Tilg $\mathrm{H}$, Adolph TE, Gerner RR, et al. The intestinal microbiota in colorectal cancer. Cancer Cell 2018;33:954-64.

8 Wong SH, Zhao L, Zhang X, et al. Gavage of fecal samples from patients with colorectal cancer promotes intestinal carcinogenesis in germ-free and conventional mice. Gastroenterology 2017:153:1621-33.

9 Kostic AD, Chun E, Robertson L, et al. Fusobacterium nucleatum potentiates intestina tumorigenesis and modulates the tumor-immune microenvironment. Cell Host Microbe 2013;14:207-15.

10 Rubinstein MR, Wang X, Liu W, et al. Fusobacterium nucleatum promotes colorecta carcinogenesis by modulating E-cadherin/ $\beta$-catenin signaling via its FadA adhesin. Cell Host Microbe 2013;14:195-206.

11 Yu T, Guo F, Yu Y, et al. Fusobacterium nucleatum promotes chemoresistance to colorectal cancer by modulating autophagy. Cell 2017;170:548-63. e16.

12 Tsoi H, Chu ESH, Zhang $\mathrm{X}$, et al. Peptostreptococcus anaerobius induces intracellular cholesterol biosynthesis in colon cells to induce proliferation and causes dysplasia in mice. Gastroenterology 2017;152:1419-33.

13 Liang Q, Chiu J, Chen Y, et al. Fecal bacteria act as novel biomarkers for noninvasive diagnosis of colorectal cancer. Clin Cancer Res 2017;23:2061-70.

14 Xie Y-H, Gao Q-Y, Cai G-X, et al. Fecal Clostridium symbiosum for noninvasive detection of early and advanced colorectal cancer: test and validation studies. EBioMedicine 2017;25:32-40.

15 Shah MS, DeSantis TZ, Weinmaier T, et al. Leveraging sequence-based faecal microbial community survey data to identify a composite biomarker for colorectal cancer. Gut 2018;67:882-91.

16 Lee JK, Liles EG, Bent S, et al. Accuracy of fecal immunochemical tests for colorecta cancer: systematic review and meta-analysis. Ann Intern Med 2014;160:171.
17 Robertson DJ, Lee JK, Boland CR, et al. Recommendations on fecal immunochemical testing to screen for colorectal neoplasia: a consensus statement by the US MultiSociety Task force on colorectal cancer. Gastroenterology 2017;152:1217-37.

18 Yutin N, Galperin MY. A genomic update on clostridial phylogeny: gram-negative spore formers and other misplaced clostridia. Environ Microbiol 2013;140:2631-41.

19 Traore SI, Azhar El, Yasir M, et al. Description of 'Blautia phocaeensis' sp. nov. and 'Lachnoclostridium edouardi' sp. nov., isolated from healthy fresh stools of Saudi Arabia Bedouins by culturomics. New Microbes New Infect 2017;19:129-31.

20 Pham T-P-T, Cadoret F, Alou MT, et al. 'Urmitella timonensis' gen. nov., sp. nov., 'Blautia marasmi' sp. nov., 'Lachnoclostridium pacaense' sp. nov., 'Bacillus marasmi' sp. nov. and 'Anaerotruncus rubiinfantis' sp. nov., isolated from stool samples of undernourished African children. New Microbes New Infect 2017;17:84-8.

21 Tidjani Alou M, Khelaifia S, La Scola B, et al. "Lachnoclostridium touaregense," a new bacterial species isolated from the human gut microbiota. New Microbes New Infect 2016;14:51-2

22 Zhang S, Cai S, Ma Y. Association between Fusobacterium nucleatum and colorectal cancer: progress and future directions. J Cancer 2018;9:1652-9.

23 Flanagan L, Schmid J, Ebert M, et al. Fusobacterium nucleatum associates with stages of colorectal neoplasia development, colorectal cancer and disease outcome. Eur J Clin Microbiol Infect Dis 2014:33:1381-90.

24 Amitay EL, Werner S, Vital M, et al. Fusobacterium and colorectal cancer: causal factor or passenger? Results from a large colorectal cancer screening study. Carcinogenesis 2017;38:781-8.

25 Watanabe Y, Nagai F, Morotomi M, et al. Bacteroides clarus sp. nov., Bacteroides fluxus sp. nov. and Bacteroides oleiciplenus sp. nov., isolated from human faeces. Int $\mathrm{J}$ Syst Evol Microbiol 2010;60:1864-9.

26 Steer T, Collins MD, Gibson GR, et al. Clostridium hathewayi sp. nov., from human faeces. Syst App/ Microbiol 2001;24:353-7.

27 Imperiale TF, Ransohoff DF, Itzkowitz SH, et al. Multitarget stool DNA testing for colorectal-cancer screening. N Eng/ J Med 2014:370:1287-97.

28 Jalanka J, Salonen A, Salojärvi J, et al. Effects of bowel cleansing on the intestinal microbiota. Gut 2015;64:1562-8.

29 Nakatsu G, Zhou H, Wu WKK, et al. Alterations in enteric virome are associated with colorectal cancer and survival outcomes. Gastroenterology 2018;155:529-41.

30 Coker 00, Nakatsu G, Dai RZ, et al. Enteric fungal microbiota dysbiosis and ecological alterations in colorectal cancer. Gut 2019;68:654-62.

31 Li H, Durbin R. Fast and accurate short read alignment with Burrows-Wheeler transform. Bioinformatics 2009;25:1754-60.

32 Morgulis A, Gertz EM, Schäffer AA, et al. A fast and symmetric dust implementation to mask low-complexity DNA sequences. J Comput Biol 2006;13:1028-40.

33 Quinlan AR, Hall IM. BEDTools: a flexible suite of utilities for comparing genomic features. Bioinformatics 2010;26:841-2.

34 Franzosa EA, Mclver LJ, Rahnavard G, et al. Species-level functional profiling of metagenomes and metatranscriptomes. Nat Methods 2018;15:962-8.

35 Seemann T. Prokka: rapid prokaryotic genome annotation. Bioinformatics 2014:30:2068-9.

36 Wong SH, Kwong TNY, Chow T-C, et al. Quantitation of faecal Fusobacterium improves faecal immunochemical test in detecting advanced colorectal neoplasia. Gut 2017;66:1441-8

37 DeLong ER, DeLong DM, Clarke-Pearson DL. Comparing the areas under two o more correlated receiver operating characteristic curves: a nonparametric approach. Biometrics 1988:44:837-45.

38 Youden WJ. Index for rating diagnostic tests. Cancer 1950;3:32-5. 